\title{
Estimation of the Mean Droplet Diameters for an Ultrasonically Atomized Spray
}

\author{
Ruben Carlos Esquivel ${ }^{*}$, José Eduardo Ramírez, William Vicente \\ Instituto de Ingeniería, Universidad Nacional Autónoma de México, Mexico City, Mexico \\ ${ }^{*}$ Corresponding author email: REsquivelH@iingen.unam.mx
}

\begin{abstract}
The calculation of the spray characteristics from the physical parameters that model the atomization process is required to develop atomizers for practical applications. In this paper, a model for the thin-film ultrasonic atomization and its dimensionless numbers are presented, the model is based on the capillary wave theory enhanced by cavitation. Experimental distributions of high-frequency atomized sprays are sampled for aqueous ethanol and aqueous glycerine mixtures using the immersion method. Correlations for the number, surface, volume, and Sauter mean diameters based on the dimensionless numbers are fitted and validated from the experimental data.
\end{abstract}

\section{Keywords}

Ultrasonic Atomization; Thin-Film Atomizer; Mean Diameter.

\section{Introduction}

Ultrasonic atomization is the fragmentation of a bulk liquid body into droplets by the action of high-frequency and high-intensity acoustic waves. The atomization process takes place when the free surface of a liquid body deposited over a substrate is being radiated by an acoustic wave produced by the vibration of the substrate. Droplets of uniform sizes are ejected at low speeds from the free surface. The main advantage of ultrasonic atomization is the ability to control the size and flow rate of the produced spray from the physical parameters that govern the atomization process [1]. The design of ultrasonic atomizers for practical applications requires analytical tools that predict the spray characteristics from the liquid physical properties and the parameters that govern the atomization process.

The mathematical description of a spray consists of the size distribution and the speed distribution of the spray's droplets. The moments of the droplet-size distribution are a measurement of the spray performance in different applications, such as mass transfer, spray drying, chemical reactions, and combustion [2].

Based on experimental observations of the ultrasonic atomization process, Lang concluded that the droplets are ejected from the free surface by standing capillary waves produced by the action of the acoustic wave [3]. Lang proposed that the number median diameter of the spray can be estimated as a fraction of the wavelength of the surface waves. This idea has been further developed to estimate different mean diameters from the wavelength of the capillary waves.

Sindayihebura et al. (1997) performed a stability analysis to obtain an expression for the capillary wavelength; with this expression, they fitted correlations for four mean diameters from experimental data [4]. Rajan and Pandit (2001) determined three dimensionless numbers relevant to the ultrasonic atomization and proposed to estimate the mode of the droplet-size distribution with an exponential function of the numbers as a coefficient of proportionality [5]. Avvaru et al. (2006) fitted a correlation for the number mean diameter based on the dimensionless numbers of Rajan and Pandit. They used experimental data of Newtonian and non-Newtonian fluids to obtain a correlation for fluids with pseudoplastic behaviour [6]. Barba 
et al. (2009) also employed Rajan and Pandit's dimensionless numbers to fit a correlation for the Sauter mean diameter of an alginate solution [7]. Ramisetty et al. (2013) proposed a correlation for the Sauter mean diameter using experimental data from aqueous glycerine and aqueous methanol solutions [8]. Muñoz et al. (2018) modified Rajan and Pandit's dimensionless numbers and fitted a correlation for the number mean diameter of an aqueous methanol solution [9].

In this paper, correlations to estimate the number, surface, volume, and Sauter mean diameter are fitted. The correlations are based on Lang's capillary wave hypothesis and use functions of the dimensionless numbers from a proposed atomization model to estimate the coefficient of proportionality. Experimental data of distilled water, aqueous ethanol solutions, and aqueous glycerine solutions are obtained using the immersion method to account for variations in the liquid density, viscosity, and surface tension. The atomization process is carried out at a frequency of $2.4 \mathrm{MHz}$ with variable flow rates and acoustic intensities.

\section{Ultrasonic atomization analysis}

Ultrasonic atomization occurs when an acoustic wave is directed towards the free surface of a liquid body from a vibrating substrate. On the free surface, standing capillary waves begin to grow. When the crests of the capillary waves reach a critical amplitude, they fracture releasing droplets. Figure 1 depicts the wave breakup process for thin-film atomization. In thinfilm atomization the liquid is delivered to the vibrating substrate at the same rate that it is atomized, creating a film of fluid with a constant depth. The liquid is characterized by its density $\rho$, viscosity $\mu$, and surface tension $\sigma$. The acoustic wave is characterized by the substrate's vibration amplitude $r$, which is proportional to the acoustic intensity, and the substrate's vibration frequency $f$. The standing capillary waves are characterized by their wavelength $\lambda$, and have a frequency of $f / 2$ [10]. The droplets are ejected at rate $\dot{V}$ and have a characteristic diameter $D$ that matches the diameter of a sphere of equivalent volume [11].

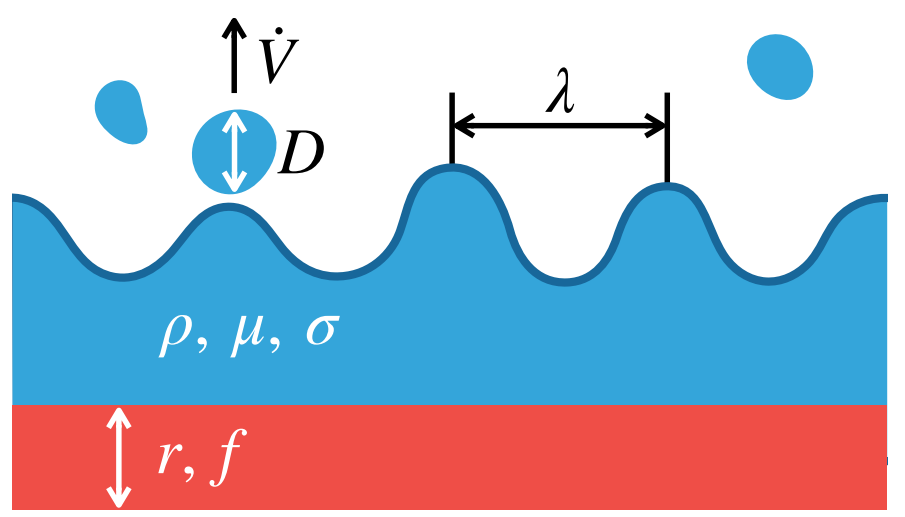

Figure 1. Illustration of the thin-film ultrasonic atomization. The vibrating substrate is illustrated in red colour; the liquid layer and the ejected droplets are illustrated in blue colour.

The Reynolds and Weber numbers are significant dimensionless numbers in the study of ultrasonic atomization [2]. The Reynolds number $R e$ represents the ratio of the oscillatory inertial force, due to the capillary waves, to viscosity:

$$
R e=\frac{\rho \dot{V}}{\mu r}
$$

The Weber number $W e$ represents the ratio of the oscillatory inertial force, due to the capillary waves, to surface tension: 


$$
W e=\frac{\rho \dot{V}^{2}}{\sigma r^{3}}
$$

The Womersley number is proposed as the third dimensionless number, which represents the ratio of the oscillatory inertial force, due to the substrate vibration, to viscosity [12]:

$$
W o=r \sqrt{\frac{\rho f}{\mu}}
$$

Following the hypothesis proposed by Lang, two correlations for the estimation of the mean diameters are proposed. In both correlations, it is assumed that a mean diameter can be estimated as a fraction of the capillary wavelength [3]. The first correlation takes the form:

$$
D_{x y}=A \Pi^{B} \lambda
$$

where $D_{x y}$ is a mean diameter, $\Pi$ is one of the abovementioned dimensionless numbers, and the coefficients A and B are fitted from experimental data. The second correlation takes the form:

$$
D_{x y}=A \Pi_{1}{ }^{B} \Pi_{2}{ }^{C} \lambda
$$

where $\Pi_{1}$ and $\Pi_{2}$ are two of the abovementioned dimensionless numbers. The three possible combinations are analysed and the coefficients $A, B$, and $C$ are fitted from experimental data for each case. In both correlations the capillary wave wavelength $\lambda$ is calculated from the following expression [13]:

$$
\lambda=4 \sqrt[8]{\frac{\mu \sigma^{2}}{\rho^{3} f^{5}}}
$$

\section{Material and Methods}

To fit the proposed correlation models, experimental data of the mean diameters are obtained for sprays through the immersion method [14].

The atomizer is shown in Figure 2 and consists of a transducer and pump. The transducer is a $12 \mathrm{~mm}$ piezoelectric PZT disk that resonates at a frequency of $2.4 \mathrm{MHz}$, the disk faces downwards to allow the sampling of droplets. The liquid is delivered to the transducer through a syringe driven by a stepper motor to maintain a steady flow rate; when steady atomization is reached, a microscope slide coated with silicon oil is placed $20 \mathrm{~mm}$ below the disk for a period of one second to collect a sample of the spray. The slide is then photographed with a Dino-Lite ${ }^{T M}$ digital microscope under a magnification of 950x. Pictures of the sample are taken for a period of thirty seconds to avoid the coalition and dissolution of droplets in the silicon oil. For each test, the sampling of droplets is repeated four times. To obtain the droplet-size distribution of the samples, the pictures are analysed using the open-source software ImageJ. To avoid measurement inaccuracies caused by the pictures of overlapping droplets, only the photographed droplets with a circularity of $90 \%$ or more are considered in the sample. The area of each droplet is measured and the diameter of a circle with an equivalent area is assigned as the diameter of the droplet, as it has been reported that the droplets captured inside the silicon oil adopt a spherical shape [15]. 

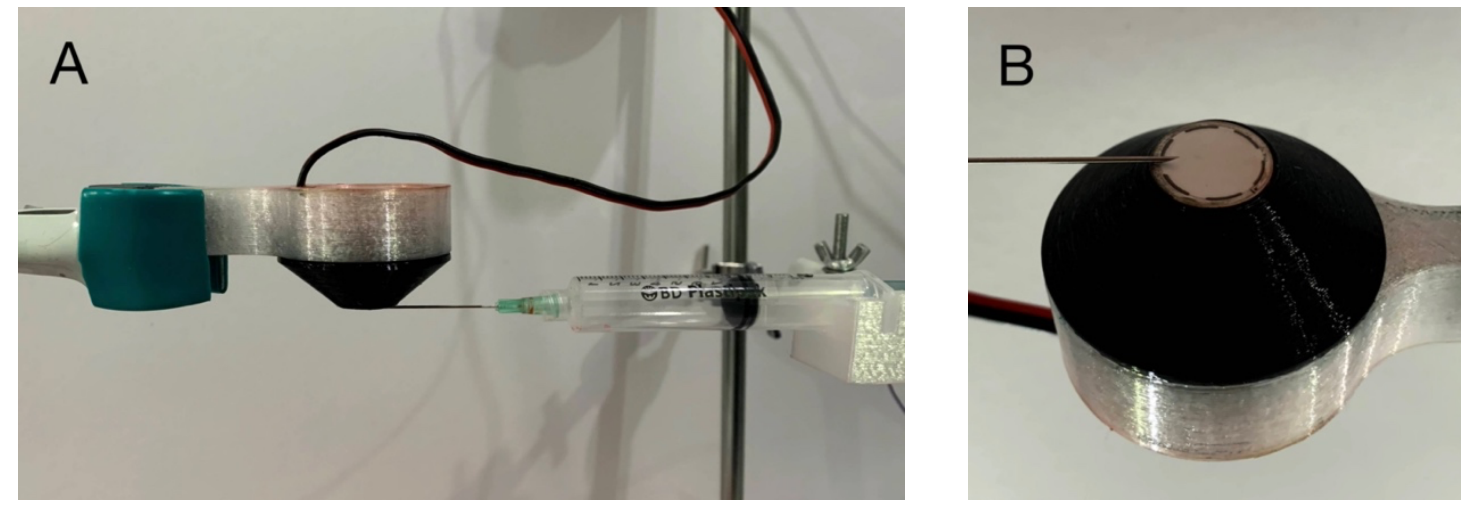

Figure 2. Ultrasonic atomizer and syringe pump. Figure A shows the atomizer arrangement during the tests.

Figure $\mathrm{B}$ shows a closeup of the piezoelectric transducer.

Aqueous ethanol and aqueous glycerine solutions are atomized with fixed flow rates and acoustic intensities to analyse the effects of the fluid density, viscosity, and surface tension. The aqueous ethanol mixtures are used to evaluate the effect of decreasing the surface tension of the liquid, while the aqueous glycerine solutions are used to evaluate the effect of increasing the viscosity of the liquid. Furthermore, to study the effects of the atomization parameters, distilled water is atomized at three different flow rates and acoustic intensities. Table 1 lists the nine tests performed. The solution's properties are obtained from published literature $[16,17]$. The vibration amplitude is evaluated by measuring the power consumed by the transducer working without any liquid at the same voltages applied during the tests, and the following relation [5]:

$$
\frac{\dot{E}}{A}=\frac{1}{2} \rho c(2 \pi r f)^{2}
$$

where $\rho$ and $c$ represent the density of air and speed of sound in air, respectively. $\dot{E}$ is the power radiated by the transducer and $A$ is the active area of the transducer, the $\dot{E}$ to $A$ ratio is the acoustic intensity at the surface of the transducer.

Table 1 - Atomization tests. The fluid properties and the atomization parameters are listed for each test. The solute percentages are expressed as mass fractions.

\begin{tabular}{c|ccccccc}
\hline Test & Solution & $\begin{array}{c}\rho \\
\mathrm{kg} / \mathrm{m}^{3}\end{array}$ & $\begin{array}{c}\mu \\
\mathrm{mPa} \mathrm{s}\end{array}$ & $\begin{array}{c}\sigma \\
\mathrm{mN} / \mathrm{m}\end{array}$ & $\begin{array}{c}f \\
\mathrm{MHz}\end{array}$ & $\begin{array}{c}r \\
\mathrm{~nm}\end{array}$ & $\begin{array}{c}\dot{V} \\
\mathrm{~mm}^{3} / \mathrm{s}\end{array}$ \\
\hline W1 & Water & 998.7 & 1.002 & 72.75 & 2.4 & 251 & 3 \\
W2 & Water & 998.7 & 1.002 & 72.75 & 2.4 & 317 & 8 \\
W3 & Water & 998.7 & 1.002 & 72.75 & 2.4 & 419 & 13 \\
E1 & Ethanol 10\% & 973.1 & 1.837 & 43.37 & 2.4 & 317 & 8 \\
E2 & Ethanol 20\% & 948.9 & 2.586 & 34.21 & 2.4 & 317 & 8 \\
E3 & Ethanol 30\% & 925.8 & 3.078 & 30.9 & 2.4 & 317 & 8 \\
G1 & Glycerine 15\% & 1032 & 1.32 & 70 & 2.4 & 317 & 8 \\
G2 & Glycerine 30\% & 1090 & 2.5 & 68 & 2.4 & 317 & 8 \\
G3 & Glycerine 50\% & 1130 & 6.05 & 65 & 2.4 & 419 & 13
\end{tabular}

\section{Results and Discussion}

The number of droplets sampled for each test and the mean diameters computed from the empirical droplet-size distributions are presented in Table 2. Bowen and Davies report that the 
number mean diameter of a spray estimated from a sample of 1500 droplets has an accuracy of $\pm 10 \%$ and an accuracy of $\pm 17 \%$ for a sample of 500 droplets [2].

Table 2 - Mean diameters and sample size. The number, surface, volume, and Sauter mean diameters are listed for each test, alongside the corresponding number of droplets sampled.

\begin{tabular}{c|ccccc}
\hline Test & Sample size & $\begin{array}{c}D_{10} \\
\mu \mathrm{m}\end{array}$ & $\begin{array}{c}D_{20} \\
\mu \mathrm{m}\end{array}$ & $\begin{array}{c}D_{30} \\
\mu \mathrm{m}\end{array}$ & $\begin{array}{c}D_{32} \\
\mu \mathrm{m}\end{array}$ \\
\hline W1 & 1790 & 9.9 & 13.4 & 17.7 & 30.9 \\
W2 & 1492 & 10.8 & 14.0 & 17.7 & 28.2 \\
W3 & 581 & 15.6 & 20.1 & 24.5 & 36.6 \\
E1 & 1571 & 9.8 & 12.5 & 15.6 & 24.3 \\
E2 & 1081 & 8.6 & 11.3 & 15.2 & 27.6 \\
E3 & 206 & 10.6 & 14.5 & 20.0 & 38.4 \\
G1 & 253 & 19.6 & 29.5 & 37.1 & 58.4 \\
G2 & 100 & 14.5 & 24.0 & 33.4 & 64.4 \\
G3 & 29 & 338.1 & 351.9 & 362.1 & 383.3
\end{tabular}

The tests performed in water show an increase in the mean diameters as the intensity and flow rate increments. The tests performed in the aqueous ethanol solutions show a decrease in the mean diameters compared to the test performed with water; according to Equation 6 , this is due to the decrease in surface tension. The tests performed in the aqueous glycerine solutions show an increase in the mean diameters compared to the test performed with water; according to Equation 6, this is due to the increase in viscosity. It should be stated that the observed atomization with the $50 \%$ glycerine solution did not produce a fog, unlike the other analysed solutions; instead, it ejected visible droplets that were manually measured from the photographs. Due to the size of these droplets, they may not adopt a spherical shape inside the silicon oil, therefore not being an accurate measurement of the droplet size. The $15 \%$ and $30 \%$ glycerine solutions both produced fog and ejected visible particles, correspondingly, the measured mean diameters of these solutions are significantly larger. For this reason, the aqueous glycerine solutions tests are excluded from the regression analysis to fit the correlations. The atomization of the glycerine solutions may occur under a different process than the rest of the solutions.

The first and second models are fitted with the Reynolds, Weber, and Womersley numbers. The accuracy of the correlations is measured using the relative standard error (RSE), and the two samples Student's $t$ and Fisher's $F$ tests. The RSE is a measure of the mean relative error of the correlations [18]. The Student's $t$ and Fisher's $F$ tests evaluate the critical confidence required to accept that the estimated samples and the experimental samples come from the same population [19].

The number mean diameter is best estimated with the correlation fitted from the second model with the Weber and Womersley numbers:

$$
D_{10}=58.14 \cdot 10^{-3} W e^{0.0901} W o^{0.5616} \lambda
$$

The correlation has a RSE of $12 \%$, and confidences of $99.5 \%$ and $82.6 \%$ for the Student's $t$ and Fisher's $\mathrm{F}$ tests, respectively. Figure $3 \mathrm{~A}$ shows the parity plot for the four fitted models, the first model is best fitted with the Womersley number. The maximum relative error in the correlation is $14.5 \%$.

The surface mean diameter is best estimated with the correlation fitted from the second model with the Weber and Womersley numbers: 


$$
D_{20}=99.53 \cdot 10^{-3} W e^{0.0714} W o^{0.4981} \lambda
$$

The correlation has a RSE of $13.6 \%$, and confidences of $99.5 \%$ and $71.9 \%$ for the Student's $t$ and Fisher's $\mathrm{F}$ tests, respectively. Figure 3B shows the parity plot for the four fitted models, the first model is best fitted with the Womersley number. The maximum relative error in the correlation is $16.8 \%$.

The volume mean diameter is best estimated with the correlation fitted from the second model with the Womersley and Reynolds numbers:

$$
D_{30}=36.47 W o^{0.6607} R e^{-0.2047} \lambda
$$

The correlation has a RSE of $14.8 \%$, and confidences of $99.9 \%$ and $46.9 \%$ for the Student's $t$ and Fisher's $\mathrm{F}$ tests, respectively. Figure $3 \mathrm{C}$ shows the parity plot for the two fitted models, the first model is best fitted with the Womersley number. The maximum relative error in the correlation is $20.4 \%$. The low confidence of the Fisher's $F$ test means that this expression does not estimate accurately the volume mean diameter.

The Sauter mean diameter is best estimated with the correlation fitted from the second model with the Womersley and Reynolds numbers:

$$
D_{32}=136.48 W o^{0.5033} R^{-0.3035} \lambda
$$

The correlation has a RSE of $18.1 \%$, and confidences of $99.8 \%$ and $3.6 \%$ for the Student's $t$ and Fisher's $\mathrm{F}$ tests, respectively. Figure 3D shows the parity plot for the two fitted models. The maximum relative error in the correlation is $21.7 \%$. The low confidence of the Fisher's $F$ test means that this expression does not estimate accurately the Sauter mean diameter.
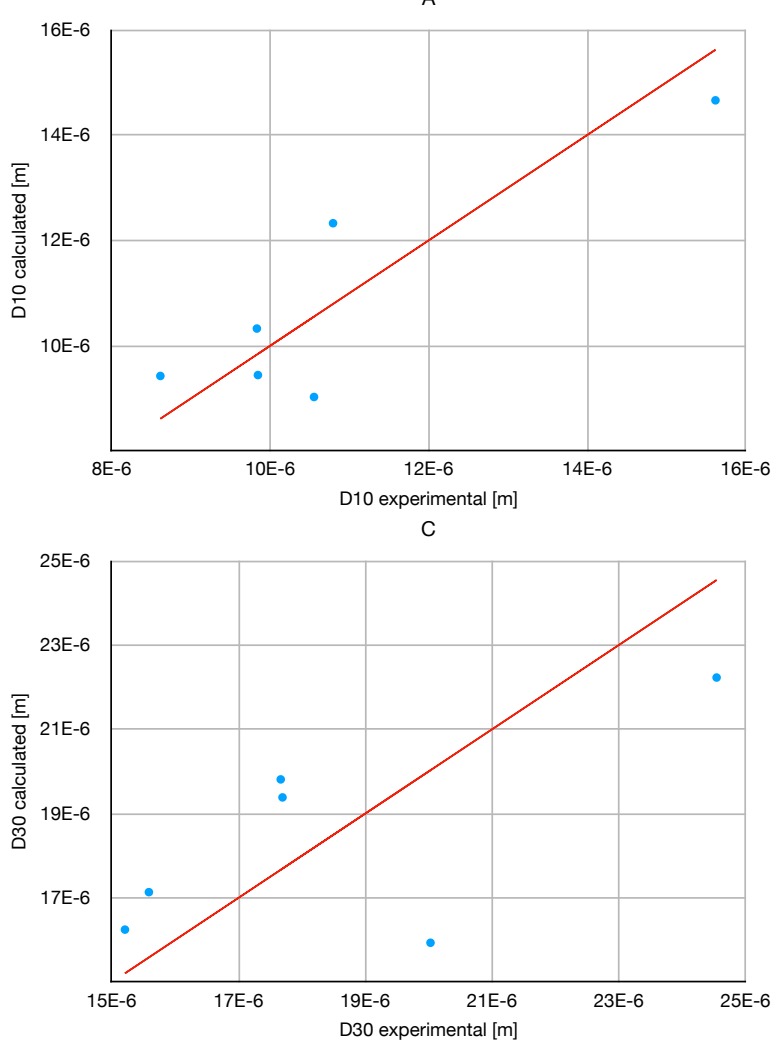
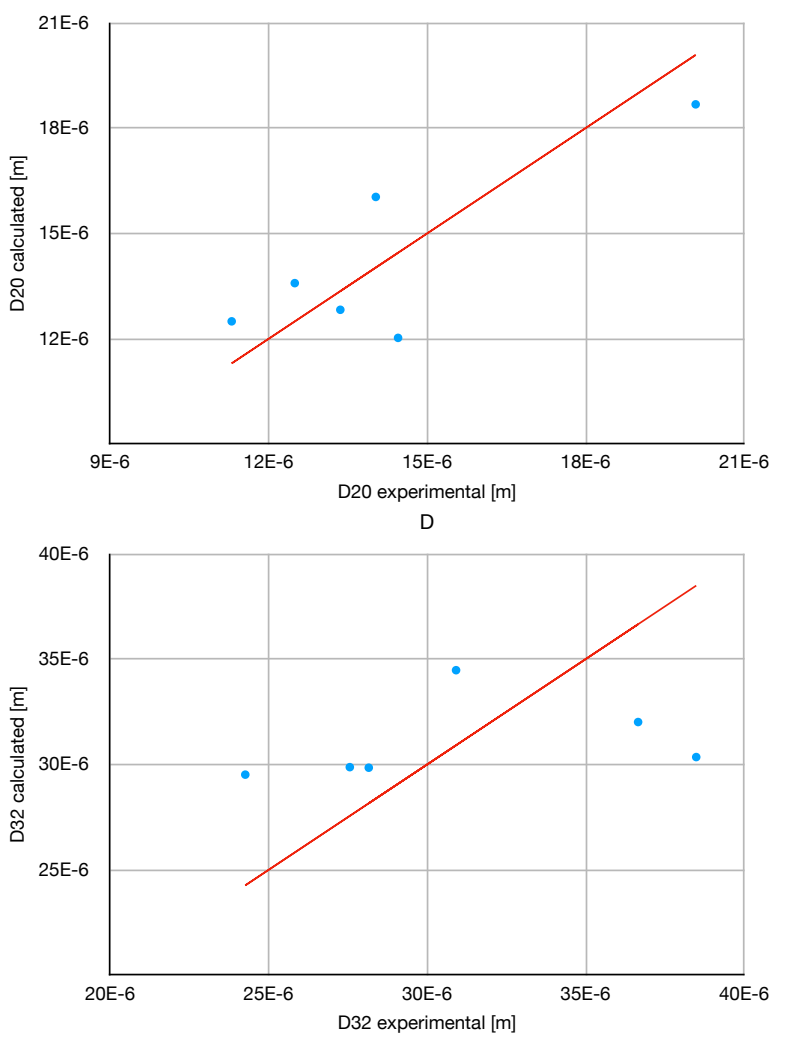

Figure 3. Parity plots of the four fitted correlations for each mean diameter: Figure A depicts the number mean diameter, Figure B depicts the surface mean diameter, Figure $\mathrm{C}$ depicts the volume men diameter, and Figure D depicts the Sauter mean diameter. 


\title{
Conclusions
}

Correlations to estimate the number, surface, volume and Sauter mean diameters were developed from the dimensionless numbers of a proposed model of ultrasonic atomization. The correlations consider the properties of the atomized fluid and the parameters of the atomization process and agree with high confidence with the experimental values. The number and surface mean diameters are best estimated with the Weber and Womersley dimensionless numbers. The volume and Sauter mean diameters are not accurately estimated with the developed correlations. A possible explanation is that the sample size did not provide reliable measurements of the volume and Sauter mean diameters.

The size of the mean diameters for the sprays atomized with glycerine solutions, and the observed ejected droplets from the experimental tests, suggest that the increase in density and viscosity may affect the main process involved in the atomization. Cavitation or inertial forces due to substrate vibration may be the dominant process in the aqueous glycerine solutions instead of the capillary waves for the aqueous ethanol solutions.

To develop a better understanding of the atomization process and the relationship of the atomization parameters to the obtained spray, more experimental tests that explore the effects of the atomization parameters and the fluid properties on the mean diameters are desirable.

\section{Acknowledgments}

The authors acknowledge the Engineering Institute (II) and the Emissions Research and Control Unit (UNICE) for the support provided in the development of the present research.

The authors acknowledge the financial support provided by the National Autonomous University of Mexico (UNAM) through the projects AG101018 and PE108418.

The authors acknowledge the National Council of Science and Technology (CONACYT) for the grants 721385 and 721437 .

\author{
Nomenclature \\ $\rho \quad$ density $\left[\mathrm{kg} / \mathrm{m}^{3}\right]$ \\ $\mu \quad$ viscosity [mPa s] \\ $\sigma \quad$ surface tension $[\mathrm{mN} / \mathrm{m}]$ \\ $c \quad$ speed of sound in air $[\mathrm{m} / \mathrm{s}]$ \\ $\lambda \quad$ capillary wavelength $[\mu \mathrm{m}]$ \\ $f \quad$ frequency $[\mathrm{MHz}]$ \\ $r \quad$ vibration amplitude $[\mathrm{nm}]$ \\ $\dot{V} \quad$ flow rate $\left[\mathrm{mm}^{3} / \mathrm{s}\right]$ \\ $\dot{E} \quad$ power radiated by the transducer [W] \\ $A \quad$ active area of the transducer [ $\mathrm{mm}^{2}$ ] \\ $D_{x y} \quad$ undefined mean diameter $[\mu \mathrm{m}]$ \\ $D_{10} \quad$ number mean diameter [ $\left.\mu \mathrm{m}\right]$ \\ $D_{20} \quad$ surface mean diameter $[\mu \mathrm{m}]$ \\ $D_{30} \quad$ volume mean diameter $[\mu \mathrm{m}]$ \\ $D_{32} \quad$ Sauter mean diameter $[\mu \mathrm{m}]$ \\ Re Reynolds number \\ We Weber number \\ Wo Womersley number
}




\section{References}

[1] Ashokkumar, M., Cavalieri, F., Chemat, F., Kenji, O., Sambandam, A., Yasui, K., Zisu, B., 2016, "Handbook of Ultrasonics and Sonochemistry."

[2] Lefebvre, A. H., and McDonell, V. G., 2017, "Atomization and Sprays."

[3] Lang, R. J., 1962, The Journal of the Acoustical Society of America, 34 (1), pp. 6-8.

[4] Sindayihebura, D., Cousin, J., Dumouchel, C., 1997, Particle and Particle Systems Characterization, 14 (2), pp. 93-101.

[5] Rajan, R., and Pandit, A. B., 2001, Ultrasonics, 39 (4), pp. 235-255.

[6] Avvaru, B., Patil, M. N., Gogate, P. R., Pandit, A. B., 2006, Ultrasonics, 44 (2), pp. 146158.

[7] Barba, A. A., d'Amore, M., Cascone, S., Lamberti, G., Titomanlio, G., 2009, Chemical Engineering and Processing, 48 (10), pp. 1477-1483.

[8] Ramisetty, K. A., Pandit, A. B., Gogate, P. R., 2013, Ultrasonics Sonochemistry, 20 (1), pp. 254-264.

[9] Muñoz, M. I., Goutier, S., Foucaud, S., Mariaux, G., Poirier, T., 2018, Ultrasonics, 84, pp. 25-33.

[10] Müller, H. W., Friedrich, R., Papathanassiou, D., 1998, "Evolution of Spontaneous Structures in Dissipative Continuous Systems."

[11] ASTM E799-03 (2015).

[12] Garih, H., Julius, S., Estivalezes, J. L., Casalis, G., 2017, Phys. Fluids, 29, 104103.

[13] Esquivel Hernandez, R. C., 2020, MS. Thesis, UNAM.

[14] Fujimatsu, T., Kito, M., Kondo, K., 2014, WIT Transactions on Engineering Sciences, 82, pp. 191-202.

[15] Tate, R. W., 1961, A.I.Ch.E. Journal, 7 (4), pp. 574-577.

[16] Khattab, I.S., Bandarkar, F., Fakhree, M.A.A., Jouyban, A., 2012, Korean Journal of Chemical Engineering, 29, pp. 812-817.

[17] Takamura, K., Fischer, H., Morrow, N. R., 2012, Journal of Petroleum Science and Engineering, 98-99, pp. 50-60.

[18] OFR 40 CFR § 136.6, 2020.

[19] NIST, 2013 "Engineering Statistics Handbook." 